\title{
Synthesis and Host-Guest Studies of Chiral
}

\section{$N$-linked Peptidoresorc[4]arenes}

\author{
Bruno Botta, ${ }^{* \dagger}$ Ilaria D'Acquarica, ${ }^{\dagger}$ Giuliano Delle Monache,${ }^{\dagger}$ Deborah Subissati ${ }^{\dagger}$ \\ Gloria Uccello-Barretta, ${ }^{*}$ Massimo Mastrini, ${ }^{*}$ Samuele Nazzi, ${ }^{*}$ Maurizio Speranza ${ }^{* \dagger}$
}

Dip. di Studi di Chimica e Tecnologia delle Sostanze Biologicamente Attive, Università "La Sapienza", P.le Aldo Moro 5, 00185 Roma (Italy); Dipartimento di Chimica e Chimica Industriale, Università di Pisa, Via Risorgimento 35, 56125 Pisa, Italy

bruno.botta@uniroma1.it; gub@dcci.unipi.it; maurizio.speranza@uniroma1.it

\section{SUPPORTING INFORMATION AVAILABLE}

Data used for the determination of hetero-association constants by the Foster-Fyfe method for complexes [10•6] and [10•ent-6] (Tables $1 \mathrm{~S}$ and 2S). General Experimental Methods. Characterization of dipeptide methyl esters 6, 9, ent-6, ent-9. ${ }^{1} \mathrm{H}$ and ${ }^{13} \mathrm{C}$ NMR signals of peptidoresorc[4]arene 11 and ${ }^{1}$ H NMR spectra of peptidoresorc[4]arenes $\mathbf{1 0}$ and 11. Total pages: 7.

\footnotetext{
† Università degli Studi di Roma "La Sapienza"; phone: +39.06.49912781.

† Università di Pisa.
} 
Table 1S. Data used for the determination of the hetero-association constant by the Foster-Fyfe method: $\Delta \delta_{\text {obs }}$ is the observed chemical shift variation $\left(\delta_{\text {mix }}-\delta_{\mathrm{f}}\right)$ for the $\mathrm{NH}$ proton of $\mathbf{6}(0.1 \mathrm{mM})$ in the presence of an increasing molar excess of $\mathbf{1 0}$ (from $7 \mathrm{mM}$ to $16 \mathrm{mM}$ ) and [10] is the total concentration of the peptidoresorc[4]arene.

\begin{tabular}{ll}
\hline$\Delta \delta_{\text {obs }} /[\mathbf{1 0}]$ & $\Delta \delta_{\text {obs }}$ \\
\hline 100290 & 401.2 \\
59466 & 416.3 \\
4247 & 423.5 \\
33190 & 431.5 \\
27342 & 437.5 \\
\hline
\end{tabular}

Table 2S. Data used for the determination of the hetero-association constant by the Foster-Fyfe method: $\Delta \delta_{\text {obs }}$ is the observed chemical shift variation $\left(\delta_{\text {mix }}-\delta_{\mathrm{f}}\right)$ for the NH proton of ent-6 $(0.3 \mathrm{mM})$ in the presence of an increasing molar excess of $\mathbf{1 0}$ (from $7 \mathrm{mM}$ to $16 \mathrm{mM}$ ) and [10] is the total concentration of the peptidoresorc[4]arene.

\begin{tabular}{ll}
\hline$\Delta \delta_{\text {obs }} /[\mathbf{1 0}]$ & $\Delta \delta_{\text {obs }}$ \\
\hline-62943 & -440.6 \\
-33070 & -330.7 \\
-21077 & -274.00 \\
-9625.0 & -154.00 \\
\hline
\end{tabular}


General Experimental Methods. Melting points (Mp) are uncorrected. ${ }^{1} \mathrm{H}$ and ${ }^{13} \mathrm{C}$ NMR spectra were run at $400 \mathrm{MHz}$ and $75 \mathrm{MHz}$, respectively $\left(\mathrm{TMS}=0 \mathrm{ppm}\right.$ as internal standard, in $\mathrm{CDCl}_{3}$ solutions, unless otherwise reported), and also at $600 \mathrm{MHz}$ and $150 \mathrm{MHz}$ respectively, using a $5 \mathrm{~mm}$ broadband inverse probe with z-axis gradient. The sample temperature was maintained at $298 \mathrm{~K}$. DOSY experiments were carried out by using a stimulated echo sequence with self-compensating gradient schemes, a spectral width of $8000 \mathrm{~Hz}$ and $64 \mathrm{~K}$ data points. Typically, a value of $200 \mathrm{~ms}$ was used for $\Delta$, $1.0 \mathrm{~ms}$ for $\delta$, and $\mathrm{g}$ was varied in 30 steps (16 transients each) to obtain an approximately $90-95 \%$ decrease in the resonance intensity at the largest gradient amplitudes. The baselines of all arrayed spectra were corrected prior to processing the data. After data acquisition, each FID was apodized with 1.0 Hz line broadening and Fourier transformed. The data were processed with the DOSY macro (involving the determination of the resonance heights of all the signals above a pre-established threshold and the fitting of the decay curve for each resonance to a Gaussian function), to obtain pseudo two dimensional spectra with NMR chemical shifts along one axis and calculated diffusion coefficients along the other. Proton 1D ROESY spectra were recorded using selective pulses generated by means of the Varian Pandora Software. The selective 1D ROESY spectra were acquired with 1024 scans in $32 \mathrm{~K}$ data points with a $5 \mathrm{~s}$ relaxation delay and a mixing time ranging of $0.5 \mathrm{~s}$.

Mass spectra (MS) were obtained on a ion-trap mass spectrometer with an electrospray ionization (ESI) source, equipped with a nitrogen generator. Conditions as follows: source voltage $=+5.0 \mathrm{kV}$; sheath gas $=25 \mathrm{AU}$ (Arbitrary Units); auxiliary gas $=10 \mathrm{AU}$; capillary voltage $=+10.0 \mathrm{~V}$; capillary temperature $=180{ }^{\circ} \mathrm{C}$; tube lens offset $=+15 \mathrm{~V}$. 
L-Valyl-L-leucine methyl ester, TFA salt (6). Mp: $173-174{ }^{\circ} \mathrm{C} ;{ }^{1} \mathrm{H}$ NMR $\left(400 \mathrm{MHz}, \mathrm{MeOH}-d_{4}, 298\right.$

$\mathrm{K}): \delta 4.90\left(\mathrm{~s}, 3 \mathrm{H}, \mathrm{NH}_{3}{ }^{+}\right) ; 4.50(\mathrm{t}, 1 \mathrm{H}, \mathrm{CH}$, leucine $) ; 3.74(\mathrm{~d}, 1 \mathrm{H}, \mathrm{NH}) ; 3.71(\mathrm{~s}, 3 \mathrm{H}, \mathrm{OMe}) ; 3.31(\mathrm{~d}, 1 \mathrm{H}$, $\mathrm{CH}$, valine); 2.24 (m, 1H, CH iPr); $1.73\left(\mathrm{~m}, 1 \mathrm{H}, \mathrm{CH}\right.$ iBu); 1.66 (m, 2H, $\left.\mathrm{CH}_{2} \mathrm{iBu}\right) ; 1.09$ (d, 3H, Me); 1.07 (d, 3H, Me); 0.97 (d, 3H, Me); 0.94 (d, 3H, Me). ${ }^{13} \mathrm{C}$ NMR (75 MHz, MeOH-d, $\left.298 \mathrm{~K}\right): \delta 172.6$ $(\mathrm{C}=\mathrm{O}$ ester $) ; 168.4(\mathrm{C}=\mathrm{O}$ amide $) ; 58.1(\mathrm{CH}-\mathrm{N}$ valine $) ; 51.9(\mathrm{OMe}) ; 51.3(\mathrm{CH}-\mathrm{N}$ leucine $) ; 39.8\left(\mathrm{CH}_{2}\right.$ iBu); 30.3 (CH iPr); $24.4(\mathrm{CH} \mathrm{iBu}) ; 22.8(\mathrm{Me}) ; 20.8$ (Me); $17.4(\mathrm{Me}) ; 16.9(\mathrm{Me})$. ESI-MS (pos.): $\mathrm{m} / z$ found $245.0\left([M+\mathrm{H}]^{+}\right), \mathrm{C}_{12} \mathrm{H}_{24} \mathrm{~N}_{2} \mathrm{O}_{3}$ (free base) requires 244.33 .

L-Leucyl-L-valine methyl ester, TFA salt (9). Mp: $172-173{ }^{\circ} \mathrm{C} .{ }^{1} \mathrm{H}$ NMR $\left(400 \mathrm{MHz}, \mathrm{MeOH}-d_{4}, 298\right.$ $\mathrm{K}): \delta 4.90\left(\mathrm{~s}, 3 \mathrm{H}, \mathrm{NH}_{3}^{+}\right) ; 4.36(\mathrm{~d}, 1 \mathrm{H}, \mathrm{NH}) ; 4.00(\mathrm{t}, 1 \mathrm{H}, \mathrm{CH}$, leucine $) ; 3.72(\mathrm{~s}, 3 \mathrm{H}, \mathrm{OMe}) ; 3.31(\mathrm{~d}, 1 \mathrm{H}$, $\mathrm{CH}$, valine); 2.24 (m, 1H, $\mathrm{CH}$ iPr); $1.72\left(\mathrm{~m}, 2 \mathrm{H}, \mathrm{CH}_{2} \mathrm{iBu}\right) ; 1.64$ (m, 1H, $\mathrm{CH}$ iBu); 0.99 (d, 12H, $\left.\mathrm{CH}_{3}\right)$. ${ }^{13} \mathrm{C}$ NMR $\left(75 \mathrm{MHz}, \mathrm{MeOH}-d_{4}, 298 \mathrm{~K}\right): \delta 171.5(\mathrm{C}=\mathrm{O}$ ester); $169.6(\mathrm{C}=\mathrm{O}$ amide); $58.2(\mathrm{CH}-\mathrm{N}$ valine); $51.9(\mathrm{OMe}) ; 51.7$ (CH-N leucine); $40.3\left(\mathrm{CH}_{2} \mathrm{iBu}\right) ; 30.4$ (CH iPr); $24.0(\mathrm{CH}$ iBu); $21.9(\mathrm{Me}) ; 21.1$ (Me); 18.3 (Me); 17.4 (Me). ESI-MS (pos.): $m / z$ found $245.0\left([M+\mathrm{H}]^{+}\right), \mathrm{C}_{12} \mathrm{H}_{24} \mathrm{~N}_{2} \mathrm{O}_{3}$ (free base) requires 244.33.

D-Valyl-D-leucine methyl ester, TFA salt (ent-6). ${ }^{1} \mathrm{H} \mathrm{NMR},{ }^{13} \mathrm{C} \mathrm{NMR}$, and ESI-MS data are coincident with those reported for 6 .

D-Leucyl-D-valine methyl ester, TFA salt (ent-9). ${ }^{1} \mathrm{H}$ NMR,${ }^{13} \mathrm{C} \mathrm{NMR}$, and ESI-MS data are coincident with those reported for 9. 


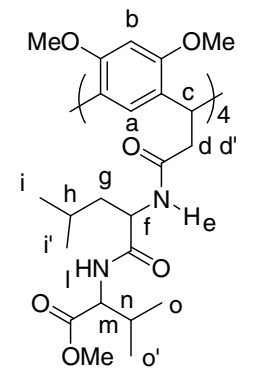

11

${ }^{1} \mathrm{H}$ NMR $\left(600 \mathrm{MHz}, \mathrm{CD}_{3} \mathrm{CN}, 298 \mathrm{~K}\right): \delta=0.60(\mathrm{~d}, \mathrm{~J}=6.7 \mathrm{~Hz}, 3 \mathrm{H}, \mathrm{Me}-\mathrm{o}), 0.78(\mathrm{~d}, \mathrm{~J}=6.7 \mathrm{~Hz}, 3 \mathrm{H}, \mathrm{Me}-$ o'), 0.83 (d, J = 6.7 Hz, 3H, Me-i), 0.88 (d, J = 6.7 Hz, 3H, Me-i'), 1.50 (m, 2H, H-g), 1.59 (m, 1H, Hh), 1.83 (m, 1H, H-n), 2.55 (dd, J = 13.9, 7.4 Hz, 1H, H-d), 2.77 (dd, J = 13.9, 7.4 Hz, 1H, H-d'), 3.63 (s, 3H, COOMe), 3.68 (s, 3H, OMe), 3.71 (s, 3H, OMe), 4.17 (t, J = 8.4 Hz, 1H, H-m), 4.27 (dt, J = 8.4, $6.8 \mathrm{~Hz}, 1 \mathrm{H}, \mathrm{H}-\mathrm{f}), 4.85$ (t, J = $7.4 \mathrm{~Hz}, 1 \mathrm{H}, \mathrm{H}-\mathrm{c}), 6.44$ (s, 1H, H-b), 6.84 (s, 1H, H-a), 6.99 (d, J = 8.4 Hz, 1H, H-l), 7.47 (d, J = 6.8 Hz, 1H, H-e); ${ }^{13} \mathrm{C}$ NMR (150 MHz, CD $\left.{ }_{3} C N, 298 K\right): \delta 173.9(\mathrm{CO}), 173.2$ (CO), $172.8(\mathrm{CO}), 157.2$ (quaternary C), 156.9 (quaternary C), $128.6(\mathrm{CH}, \mathrm{Ar}$ ), 124.4 (quaternary $\mathrm{C}$ ), 124.0 (quaternary C), $97.4(\mathrm{CH}, \mathrm{Ar}), 59.1(\mathrm{CH}), 56.8(\mathrm{OMe}), 56.4(\mathrm{OMe}), 52.5(\mathrm{OOMe}), 52.1(\mathrm{CH})$, $42.1\left(\mathrm{CH}_{2}\right), 40.9\left(\mathrm{CH}_{2}\right), 34.3(\mathrm{CH}), 31.8(\mathrm{CH}), 25.5(\mathrm{CH}), 22.9(\mathrm{Me}), 22.2(\mathrm{Me}), 19.3(\mathrm{Me}), 18.7(\mathrm{Me})$. 
${ }^{1} \mathrm{H}$ NMR $\left(600 \mathrm{MHz}, \mathrm{CDCl}_{3}, 298 \mathrm{~K}\right)$ spectrum of peptidoresorc[4] arene $\mathbf{1 0}$.

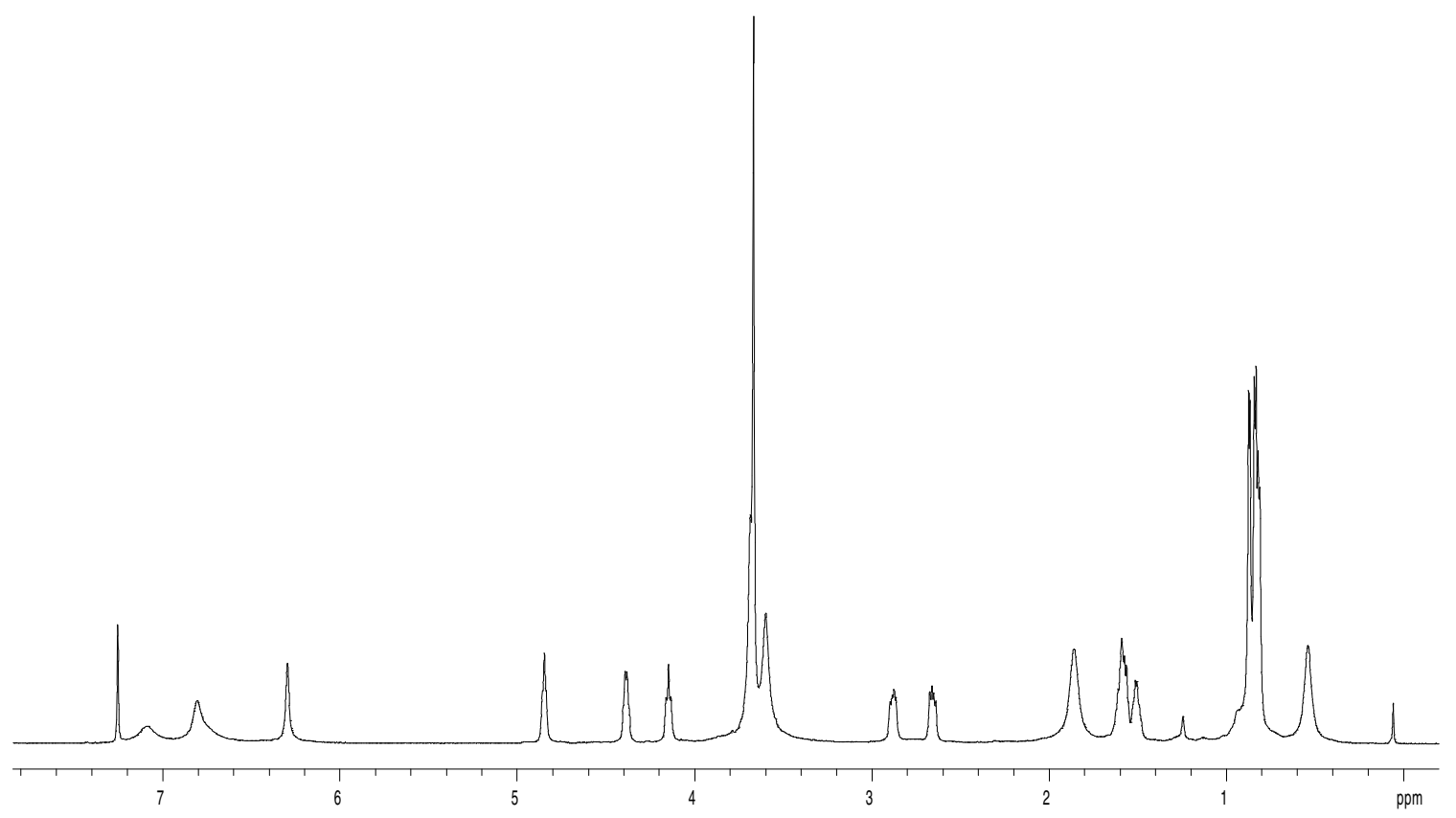


${ }^{1} \mathrm{H}$ NMR $\left(600 \mathrm{MHz}, \mathrm{CD}_{3} \mathrm{CN}, 298 \mathrm{~K}\right)$ spectrum of peptidoresorc[4]arene 11.

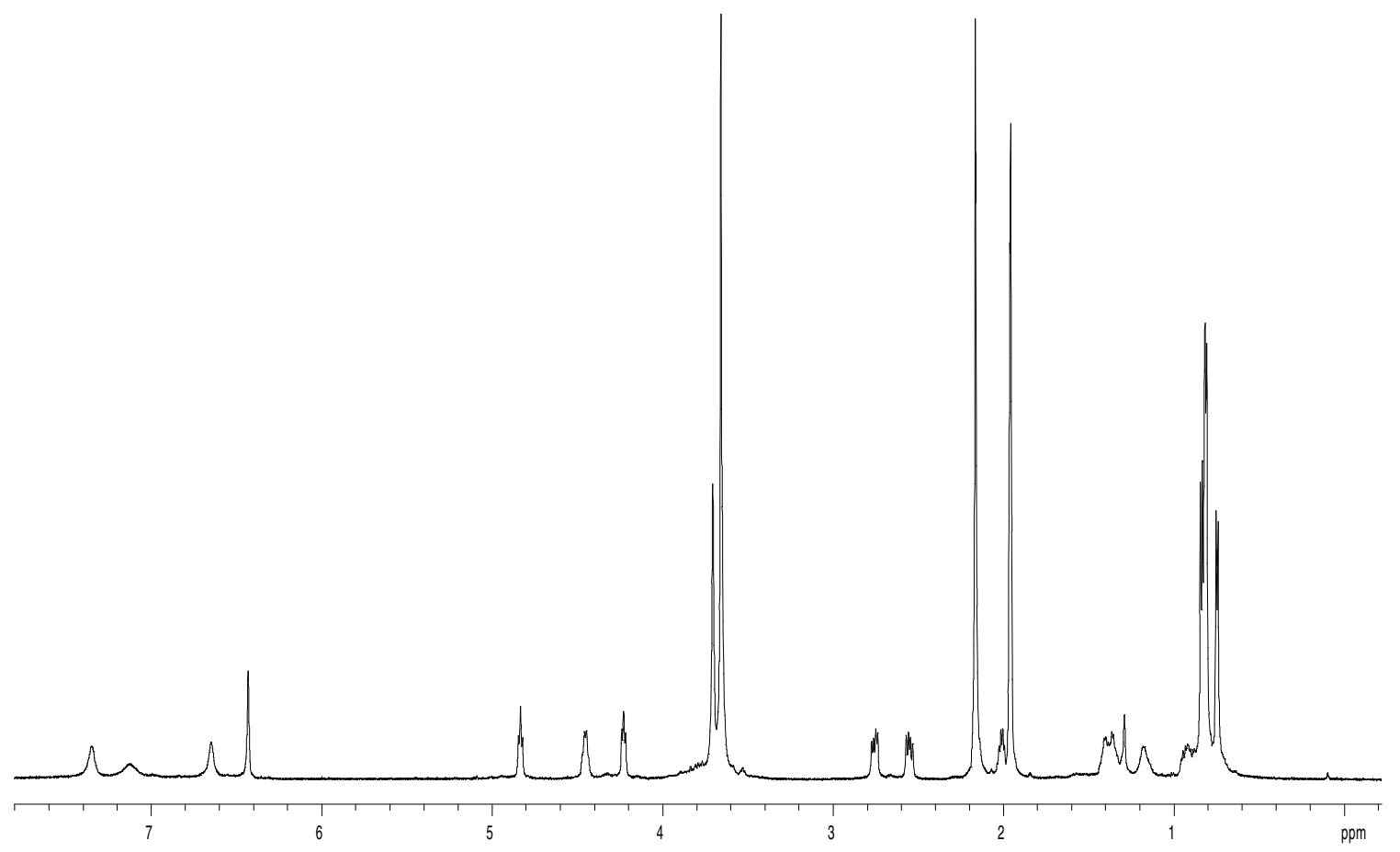

